\title{
Evaluación de herramientas digitales para la gestión del portafolio educativo
}

\author{
Montaño Flores Dolores \\ lolym19@gmail.com \\ https://orcid.org/0000-0002-6373-8139 \\ Universidad Católica del Ecuador \\ Esmeraldas-Ecuador
}

Recibido (20/12/2020), Aceptado (10/02/2021)

\begin{abstract}
Resumen: El portafolio digital educativo, es una herramienta virtual que combina recursos tecnológicos con el objeto de fortalecer los procesos de enseñanza-aprendizaje, permitiendo desarrollar criterios activos y participativos en circunstancia de docente y estudiante. La investigación describe los procesos de enseñanza- aprendizaje proponiendo nuevos sistemas pedagógicos y mejoramientos de la metodología académica. Así mismo, se proponen estrategias para contribuir con el desarrollo educativo como fuente fundamental para la solución de los graves problemas que afectan a la educación actual; además se pudo determinar que el uso de herramientas virtuales durante los procesos académicos motivará los aprendizajes de los estudiantes de forma activa, dinámica e interactiva, considerando que es de trascendental importancia que las unidades académicas, cuenten con un sistema informático que organice su documentación formativa con el propósito de administrar los procesos didácticos pedagógicos de forma óptima. Finalmente, los resultados muestran que es fundamental el uso del portafolio digital educativo, como una herramienta virtual que facilita al docente evaluar las competencias del estudiante durante el proceso de enseñanza-aprendizaje, utilizando metodologías acordes a la educación actual con la finalidad de potencializar las habilidades con criterio de desempeño.
\end{abstract}

Palabras Clave: Herramientas digitales, proceso de enseñanza-aprendizaje, portafolio digital educativo.

\section{Evaluation digital tools for the}

\section{management the portafolio educational}

\begin{abstract}
The educational digital portfolio is a virtual tool that combines technological resources in order to strengthen the teaching-learning processes, allowing the development of active and participatory criteria in teacher and student circumstances. The research describes the teaching-learning processes proposing new pedagogical systems and improvements in academic methodology. Likewise, strategies are proposed to contribute to educational development as a fundamental source for solving the serious problems that affect current education; In addition, it was determined that the use of virtual tools during the academic processes will motivate the learning of students in an active, dynamic and interactive way, considering that it is of transcendental importance that the academic units have a computer system that organizes their training documentation with the purpose of managing the pedagogical didactic processes in an optimal way. Finally, the results show that the use of the educational digital portfolio is essential, as a virtual tool that facilitates the teacher to evaluate the student's competencies during the teaching-learning process, using methodologies according to current education in order to enhance skills with performance criteria.
\end{abstract}

Keywords: Digital tools, teaching-learning process, educational digital portfolio.

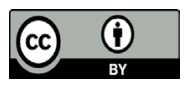




\section{I.INTRODUCCIÓN}

Los procesos de enseñanza aprendizaje tuvieron cambios trascendentales a nivel mundial, con la aparición de las nuevas Tecnologías de la Información y la comunicación (TIC), permitiendo llevar programas de enseñanza innovadores, apoyados en recursos facilitadores de la herramienta internet. Los procedimientos de evaluación docente, van a la par a los cambios educativos, en la actualidad los sistemas escolares aún mantienen la cultura de calificación y medición más que de evaluación.

De igual manera, los métodos de enseñanza aprendizaje han evolucionado a causa del mundo globalizado, desarrollando cambios sociales, educativos y radicales, enfatizándose en alternativas ajustadas en el aprendizaje de los estudiantes, donde puedan ser evaluados durante el proceso académico de forma individual y grupal, dejando a un lado sus capacidades memorísticas, centrándose en preparar al individuo en seres críticos, participativos, cooperativos y reflexivos.

Otros autores [2] afirman que el portafolio ha facilitado la organización de la información del trabajo docente, lo que generó mayor sistematicidad de la reflexión sobre su práctica educativa y promovió al uso de las tecnologías de la información y comunicación (TIC) en los procesos de planificación y evaluación, la carpeta digital misma, fue percibida por ellos como una innovación de su práctica educativa al ser valorada como una herramienta TIC novedosa y versátil.

Por otro lado, Chavarro [3] considera como estrategia de aprendizaje, el portafolio electrónico, que brinda la posibilidad de aprender del error, de mejorar el trabajo en equipo y retroalimentar permanentemente su proceso de elaboración y el logro de las competencias propias del programa de formación, lo cual está acorde con las corrientes constructivistas del aprendizaje, constituyen así las bases de los modelos pedagógicos contemporáneos.

El portafolio educativo sistematiza y organiza la información proporcionada por los docentes, agrupando un conjunto de herramientas informáticas que proporcionan la recolección de información y permitir la entrega de portafolios en el tiempo esperado.

El uso del portafolio electrónico permite monitorear los avances de los estudiantes, facilitando el seguimiento de las actividades que realizan digitalmente en clase, por ello se exige al profesorado prepararse continuamente y renovar su metodología de enseñanza, conociendo el uso adecuado de los portafolios electrónicos, ya que las TIC han llegado a la comunidad educativa de manera inesperada, además, esta preparación e integración de tecnologías, tiene como objeto concebir que los estudiantes a través de este cambio metodológico reflexionen sobre los resultados de aprendizajes generados mediante la web.

En síntesis, en relación a las teorías mostradas, las nuevas propuestas educativas en torno a la necesidad de mejorar los ambientes de enseñanza, que contribuyan a la calidad de la educación y la calidez con métodos formativos, se hace prescindible aplicar herramientas digitales que permitan mejorar los procesos de enseñanza-aprendizaje. Por lo tanto, en cumplimiento, en lo manifestado en el art.3, de los niveles de gestión del sistema de educación intercultural del Ecuador [5], se afirma que la educación es el nivel de gestión desconcentrado, encargado de asegurar la cobertura y la calidad de los servicios educativos del Distrito en todos sus niveles y modalidades, desarrollar proyectos y programas educativos, planificar la oferta educativa del Distrito Escolar, coordinar las acciones de los circuitos educativos interculturales o bilingües de su territorio y ofertar servicios con el objeto de fortalecer la gestión de la educación de forma equitativa e inclusiva, con pertinencia cultural y lingüística, que responda a las necesidades de la comunidad; cada distrito educativo intercultural y bilingüe debe corresponder al territorio definido por el Nivel Central de la Autoridad Educativa Nacional, en concordancia con el Plan Nacional de Desarrollo.

EL siguiente trabajo consistió en evaluar las herramientas digitales para la Gestión del Portafolio Educativo, como una herramienta digital necesaria para el mejoramiento de los procesos académicos y la implementación de nuevas estrategias pedagógicas para el aprendizaje. El portafolio digital es un instrumento que combina las herramientas tecnológicas con el objeto de reunir trabajos que permitan el seguimiento y la evaluación del proceso de aprendizaje del estudiante, su uso es muy útil y tiene la capacidad de incorporar las nuevas tecnologías de la información y comunicación al proceso de aprendizaje. En resumen, el portafolio digital es un fichero donde se almacenan de forma ordenada todas las planificaciones o actividades de clases durante el proceso de enseñanza-aprendizaje presentando el progreso de sus logros y resultados.

Ante lo expuesto, la evaluación de herramientas digitales, sirven de análisis en la selección del mejor recurso digital, que al ser implementado en los centros educativos facilita los beneficios de promover aprendizajes activos, desarrollar competencias en el estudiante, compartir información sin importar distancia, facilidad de acceso desde 
cualquier ordenador que se encuentre con servicio de internet.

El presente apartado se fundamentó en el conocimiento de las categorías digitales, para beneficios en el proceso de enseñanza-aprendizaje, favoreciendo el buen desenvolvimiento del docente y el desarrollo de habilidades en el estudiante. En la constitución de la república del Ecuador, literal 8 del artículo 347, se expresa la necesidad de incorporar las tecnologías de la información y comunicación en el proceso educativo y propiciar el enlace de la enseñanza con las actividades productivas o sociales.

El actual trabajo se compone de cinco secciones, la primera expone la introducción, la segunda describe el desarrollo teórico acerca de las herramientas digitales, en la tercera sección se describe la metodología utilizada para el desarrollo de los resultados, mientras que en la cuarta se exponen los resultados obtenidos en el análisis del trabajo, por último, se muestran las conclusiones planteadas en base al análisis de los resultados.

\section{II.DESARROLLO}

\section{A.Herramientas Didáctica General.}

Son cualquier tipo de soporte material o tecnológico que facilita o propicia el proceso de enseñanza-aprendizaje. Suele ser empleados por los educadores e instituciones pedagógicas o formativas, como una forma de complementar o de hacer más eficientes sus labores. En definitiva, los recursos didácticos, son mediadores para el desarrollo educativo, con el fin de elevar la calidad y eficiencia de las acciones pedagógicas [8]

Es importante señalar que las herramientas didácticas cumplen con roles importantes en la educación y rompe con los esquemas de la enseñanza convencional por lo que estimula y refuerza la firme interacción entre el estudiante y el docente, forjando flujos de conocimiento críticos y resultando así la adquisición de conocimiento de manera óptima.

\section{B.Portafolio Digital.}

Son procesos de enseñanza-aprendizaje y evaluación, que se radica en la aportación de conocimientos por parte del docente y estudiante. En ese contexto se expresa: El portafolio digital es un instrumento que combina las herramientas tecnológicas con el objeto de reunir trabajos que permitan el seguimiento y la evaluación del proceso de aprendizaje del estudiante, su uso es muy útil, y tiene la capacidad de incorporar las nuevas tecnologías de la información y comunicación al proceso de aprendizaje [6].

Se puede inferir ante lo expuesto, que es el espacio soportado por la TICS e internet, sirviendo en los procesos pedagógicos, permitiendo desarrollar criterios activos y participativos en circunstancia de docente y estudiante.

\section{Características del portafolio digital.}

El portafolio electrónico o digital permite incluir textos, gráficos o elementos de multimedia, que se pueden consultar en internet o en cualquier medio electrónico agrupa todo el material en formatos que son mucho más manejables, como CD, DVD y los distintos materiales incluidos pueden ser enlazados entre sí de modo que se puede acceder en cualquier momento a reflexiones, enlaces de interés de un determinado tema posibilita el control y la organización de los trabajos creados en los diferentes medios [9].

Cabe indicar que, a través del portafolio educativo, permite mostrar la evolución del proceso de enseñanza aprendizaje, estimulando la experiencia, reflexión e investigación del docente y estudiante.

\section{Tipos de Portafolio}

Es significante indicar, para los procesos pedagógicos, se puede usar distintos tipos de portafolio:

a)Portafolio de Evaluación: Mide el conocimiento individual en dominio de sus habilidades con carácter de desempeño, para obtener los logros alcanzados del docente y estudiante.

b)Portafolio de Aprendizaje: Permite ofrecer información sobre los objetivos del aprendizaje.

c)Portafolio de Resultado: Presenta información de los logros alcanzados a través de las mejoras en sus actividades prácticas.

d)Portafolio de Transacción: Muestra evidencia en los cambios académicos en sus diferentes niveles [10] .

Además [10] afirma que otros elementos del portafolio deben incluir el mensaje de bienvenida, la foto, el 
curriculum académico y profesional, la explicación del portafolio, y otras necesidades que se amerite como los objetivos formativos, que conforman el aprendizaje del estudiante y la base de su evaluación. Además, es importante la inclusión del desarrollo de actividades de las diferentes asignaturas la reproducción de información durante el proceso académico (páginas web, correos electrónicos, videoconferencias, otros). Otros aspectos también se consideran importantes dentro del portafolio docente, como es el diario de campo, sitio donde el estudiante anota sus reflexiones referentes a su proceso de aprendizaje y realiza su autoevaluación de las utilidades. Además deben incorporarse la Guía del Docente, que incluye las instrucciones para el desarrollo del proceso académico, como los comentarios que han dirigido el aprendizaje del estudiante.

Es oportuno, expresar que el tipo de portafolio a utilizar depende del análisis y la necesidad específica del proceso de enseñanza-aprendizaje, en nivel de conocimientos, objetivos de aprendizaje, logros o muestra de evidencias, presentando en cualquiera de los casos sus respectivos elementos y en ese marco aprovechar la herramienta para la búsqueda de mejoras en los métodos educativos.

\section{Ventajas del portafolio digital.}

El portafolio digital presenta las siguientes ventajas. A continuación se expresa:

-Permite al estudiante ser activo, crítico, cooperativo y reflexivo, ayudándolo a fijarse metas, y asumir la responsabilidad de su aprendizaje, exige el uso del internet herramienta prescindible para los procesos de enseñanza-aprendizaje.

-Versatilidad del medio digital, permitiendo almacenar información en espacios virtuales, considerando espacios informáticos que simulan procesos reales generados por un ordenador, con la capacidad de poder compartir información sin restricciones y áreas temporales.

-Almacena y conserva información del estudiante en diferentes formatos aplicados.

Los beneficios antes expuestos, fortalecen los aprendizajes educativos, dando paso a mejoras pedagógicas, potencializando nuevos esquemas curriculares.

Conviene subrayar, la importancia de definir los procesos de enseñanza aprendizaje, en el tema plataformas para el control del uso de software.

Enseñanza-aprendizaje: Es el movimiento de la actividad cognoscitiva de los estudiantes bajo la dirección del maestro, hacia el dominio de los conocimientos, las habilidades, los hábitos y la formación de una concepción científica del mundo [11].

En definitiva, los nuevos procesos pedagógicos son los intercambios de conocimientos entre dos o más sujetos, el que enseña (profesor), y el/los aprendices (estudiantes) a través de la comunicación directa o indirecta con el uso de métodos, técnicas o medios tecnológicos, con el propósito de desarrollar en el estudiante destrezas con criterio de desempeño para prepararse y enfrentar los retos del convivir diario.

\section{III.METODOLOGÍA}

Se consideraron los métodos deductivo e inductivo, el método deductivo parte de hechos generales a particulares, pudiendo afirmar que el método deductivo se fundamenta en el razonamiento que permite formular juicios partiendo de argumentos generales para demostrar, comprender o explicar los aspectos particulares de la realidad.

En ese contexto, se observaron y analizaron hechos generales, que trataron las necesidades de mejorar los procesos de enseñanza-aprendizaje de los docentes del bachillerato de la Unidad educativa Ramón Bedoya Navia y de igual manera las formas y métodos de enseñanza que utilizan los docentes particularmente.

Con respecto al método inductivo, se basa de hechos particulares a generales, se fundamenta en el razonamiento que parte de aspectos particulares para construir juicios o argumentos generales. Mediante este método se formulan las teorías y leyes científicas. El método inductivo efectúa observaciones, las ordena y clasifica, a fin de extraer conclusiones de ámbito universal partiendo del cúmulo de datos particulares. En ese marco textual, la indagación del proyecto, se orientó en analizar hechos específicos, observando los métodos y técnicas de enseñanza de los docentes de la unidad educativa Ramón Bedoya Navia de la ciudad de Esmeraldas, con el fin de sugerir estrategias pedagógicas que garanticen a los educandos impartir enseñanza de calidad y calidez.

El tipo de investigación utilizada fue descriptiva, metodología que muestra sistemáticamente las características 
de situaciones o áreas de interés, tomando en cuenta que este estudio se dirige fundamentalmente a la los fenómenos sociales o educativos en una circunstancia temporal y espacial determinada.

El uso del Portafolio Digital Educativo, está diseñado por herramientas tecnológicas facilitadas por la web, que permite almacenar las actividades desarrolladas durante el proceso de enseñanza-aprendizaje, facilitando a los educandos y al educador ser seres dinámicos, críticos reflexivos y participativos.

El universo de estudio estuvo compuesto por el nivel de bachillerato de los docentes y estudiantes de la Unidad Educativa Ramón Bedoya Navia, para realizar posteriormente el análisis de resultados. La población estuvo compuesta por dos autoridades, 25 docentes y 255 estudiantes; al ser pequeña la población de autoridades y docentes, sus datos no justifican para obtener la respectiva muestra, por lo tanto, se realizaron entrevistas a los entes mencionados, mientras que, al total de estudiantes, se aplicó la ecuación (1):

$$
\mathrm{n}=\frac{N * \&^{2} * Z^{2}}{(N-1) E^{2}+\&^{2} * Z^{2}}
$$

Siendo:

$\mathrm{N}=$ Universo o población

$\&=$ Desviación estándar de la población $(0,5)$

$\mathrm{E}=$ Límite aceptable de error $(0,9)$

$\mathrm{Z}=$ Nivel de confianza o nivel de significación $(0,95)$

Obteniendo un valor de 25,12 para la muestra de estudio.

Se utilizó un instrumento tipo cuestionario de preguntas formadas por 10 ítems, se les aplicó a los estudiantes de la entidad de estudio la cual permitió obtener la información objetiva sobre la opinión de los educandos acerca del uso de herramientas digitales que mejoren los procesos educativos. Entre los temas abordados se encuentran el nivel de satisfacción de aprendizaje, la comunicación entre docente y estudiante, el procesos de enseñanza del docente, la evaluación al desempeño docente, las tecnologías de la institución educativa, el ancho de banda de la institución, los recursos digitales para el proceso académico, el uso de herramientas digitales, el software de la documentación académica y el servicio de internet en los hogares; en la observación se empleó el instrumento de ficha de observación, permitiendo conocer y analizar la metodología utilizada por los docentes.

\section{IV.RESULTADOS}

La presente investigación tuvo como propósito estudiar y describir el uso de herramientas didácticas digitales para el proceso de enseñanza-aprendizaje, en su análisis de resultados se desarrolló un cuestionario de preguntas, formado por 10 cuestiones; el presente tratado solo se focaliza en las cuestiones más relevantes.

En la evaluación del nivel de satisfacción de los aprendizajes, el 44\% de los encuestados expresaron que el nivel de satisfacción de los aprendizajes es alto, lo que muestra en gran magnitud la preocupación en los educadores en cumplir con las exigencias ministeriales actuales. En el mismo contexto el $40 \%$ de los participantes declararon tener muy alto grado los procesos académicos utilizados por el docente. Por el contrario, solo el $12 \%$ y el $4 \%$ de los estudiantes indicaron su satisfacción de aprendizajes es medio y bajo respectivamente; expresiones que se respaldan a la falta de motivación que los docentes les transmiten durante las horas clases. Esos resultados impulsan a la necesidad de crear evaluaciones de la práctica pedagógica, siendo necesaria la creación de un plan estratégico institucional en donde se haga partícipe a los estudiantes y padres de familia en las evaluaciones del trabajo educativo, resaltando importantes aspectos como los recursos didácticos utilizados, la motivación en clases, el plan de clases, la puntualidad en tareas encomendadas, la creatividad.

En el cuestionamiento, tecnología con la que cuenta la Unidad Educativa Ramón Bedoya Navia, el 44\% de los estudiantes manifestaron que las herramientas digitales que utilizan en los procesos de enseñanza-aprendizaje es buena, gracias a la autogestión que realizan las autoridades del plantel en adquirir equipos tecnológicos en base a las necesidades educativas de la entidad educativa en mención. Por consiguiente, el $36 \%$ expresaron que es muy buena. Mientras que el 4\% expresaron que es mala; opiniones que se basan a la falta de infraestructura informática que abarque y satisfaga las necesidades educativas. En cambio, el 16\% de los educandos opinaron que la infraes- 
tructura informática de la institución educativa en referencia de estudio es excelente; referencia que promueve la necesidad de gestionar el abastecimiento de computadoras actualizadas, proyectores e internet en el laboratorio de computación, recursos tecnológicos prescindibles para el desarrollo de la institución.

En la interrogante uso de herramientas digitales, el 56\% de los encuestados expresaron hacer uso de herramientas digitales cuando se amerita. EL $24 \%$ de los encuestados declararon que a veces, en resultado de no contar con facilidades de acceso ante los recursos tecnológicos requeridos, por motivo de sus paupérrimas condiciones de vida. Contradictoriamente el $20 \%$ de los estudiantes declararon usar siempre recursos informáticos, entre esas herramientas se tiene: Teléfonos inteligentes, Tables, videocámaras, redes sociales, páginas web educativas; facilidades que permiten mejoras en sus procesos académicos.

En la pregunta software que organiza la documentación académica, el 64\% de los indagados opinaron que siempre en la U.E.R.B.N., Cuenta con un Software que organiza la documentación académica en relación a las notas de los estudiantes, opiniones basadas en el sistema informático suministrado por el Ministerio de Educación del Ecuador. Mientras que el $12 \%$ de los participantes declararon que la entidad educativa indicada conserva el sistema de notas cuando se amerita, a causa que el mencionado procedimiento no se mantiene activo permanentemente. Por el contrario, el 24\% de los estudiantes declararon que nunca administra softwares académicos (planes de clases, tareas, foros, chat, correos, calificaciones) en el docente y estudiante; expresión que amerita hacer vigente la propuesta planteada en hacer uso del portafolio digital para la gestión y práctica docente.

Con respecto a la entrevista ejecutada a los docentes de la Unidad Educativa Ramón Bedoya Navia, el 100\% de los educadores expresaron que su nivel de conocimiento en estrategias didáctica digital es intermedio, afirmación que se enmarca a las capacitaciones que concede el Ministerio de Educación del Ecuador en temas informáticos, siendo requisito para aprobar las evaluaciones que exigen en tal organismo, dando paso a una educación evolutiva acorde a niveles competitivos que requiere la sociedad educativa actual.

Resultados semejantes se presentan en estudios del uso del portafolio digital; dicho argumento [2] manifiesta los profesores han hecho reflexiones muy fructíferas tanto respecto a su planificación e interacción con los alumnos a través de los recursos digitales, dicen haber aprendido el valor de la reflexión ya que gracias a ella han revisado y reorientado su trabajo, se les han planteado nuevos retos que les han obligado a nuevos aprendizajes. Mencionan, además, estar satisfechos por haber logrado mejorar tanto los resultados académicos de los alumnos como el clima de aprendizaje [2].

\section{V.CONCLUSIONES}

Las herramientas digitales tradicionales, utilizadas por los docentes evaluados, no satisfacen el $100 \%$ de las necesidades educativas de los educandos por lo que las actividades docentes se convierten en monótonas y escasamente interactivas y poco participativas (proyector y diapositiva, correos electrónicos y pizarra).

Resulta de gran importancia que los docentes hagan uso del portafolio digital educativo, como una herramienta enriquecedora en el proceso de enseñanza. Una de las herramientas virtuales más eficientes y aptas para la gestión y práctica docente, es Eduportafolio, que facilita al docente evaluar las competencias del estudiante logradas durante el proceso de enseñanza-aprendizaje, utilizando metodologías de trabajo acorde a la educación actual.

El Eduportafolio, convertirá el área de trabajo en zonas de construcción de aprendizajes, permitiendo recopilar sus resultados y reflexiones de las diferentes actividades desarrolladas durante el proceso de enseñanza-aprendizaje dando paso a la retroalimentación en conjunto entre los participantes.

La implementación del portafolio digital educativo, motivará los aprendizajes de los estudiantes dando paso de la educación tradicional a lo trascendental, permitiendo compartir sus propios intereses, describir y autoevaluar los objetivos logrados.

\section{REFERENCIAS}

[1]D. Montaño Flores, «Repositorio Digital PUCESE,» 12 2016. [En línea]. Available: https://repositorio.pucese. edu.ec/bitstream/123456789/601/1/MONTANO\%20FLORES\%20DOLORES.pdf. [Último acceso: 253 2021]. [2]R. Rodriguez, «El portafolio digital como soporte de la practica reflexiva en la formacióndocente,» Revsiasta Iberoamericana, vol. 65, $\mathrm{n}^{\mathrm{o}}$ 18, p. 22, 2013.

[3]C. E. Chavarro Aranzalez, «El portafolio Electróinico: Una mirada desde la evaluación formativa. Caso de la institución educativa técnica Cualamaná, Melgar Tolima 2014,» 248 2015. [En línea]. Available: http://45.71.7.21/ bitstream/001/1575/1/EL\%20PORTAFOLIO\%20ELECTR\%C3\%93NICO\%20UNA\%20MIRADA\%20 
DESDE\%20LA\%20EVALUACI\%C3\%93N.pdf. [Último acceso: 223 2021].

[4]L. d. C. Hernandez, «Portafolio digital,» Centro regional de Tabasco, 135 2013. [En línea]. Available: https:// loreni1987.wordpress.com/unidad-3-portafolio-digital/1-portafolio-definiciones/. [Último acceso: 812 2015].

[5]C. d. 1. R. d. Ecuador, «Ley orgánica de educación intercultural,» 33 2017. [En línea]. Available: https://educacion.gob.ec/wp-content/uploads/downloads/2017/05/Reglamento-General-Ley-Organica-Educacion-Intercultural.pdf. [Último acceso: 183 2021].

[6]Hidalgo LLumikinga, Wilian Andrés; Moscoso Lanchang, Adolfo Alberto;, «Propuesta tecnológica del sistema E-portafolio para los docentes de la carrera de ingeniería en sistemas adminsitrativos computarizados,» 2016. [En línea]. Available: http://repositorio.ug.edu.ec/bitstream/redug/20360/1/PROYECTO\%20TECNOLOGICO\%20 DEL\%20SISTEMA\%20E-PORTAFOLIO.pdf. [Último acceso: 223 2021].

[7]C. d. 1. R. Ecuador, «Constitución del Ecuador,» 508 2008. [En línea]. Available: https://educacion.gob.ec/ wp-content/uploads/downloads/2012/08/Constitucion.pdf. [Último acceso: 1803 2021].

[8]D. Cauas, «Definición de las variables, enfoque y tipo de investigación,» 2015. [En línea]. Available: https:// scholar.google.es/scholar?hl=es\&as_sdt=0\%2C5\&q=tipo+de+investigacion+descriptiva\&btnG $=$. [Último acceso: 193 2021].

[9]J. Arevalo, «Todo acerca de portafolio digital,» Universidad Nacional de Quilmes, 239 2012. [En línea]. Available: https://jackearevalo.wordpress.com/2012/09/23/todo-acerca-del-portafolio-digital/\#. [Último acceso: 912 2015].

[10]J. Velez Loor, «Uso del portafolio para el desarrollo de habildiades comunicacionales de escritura con estudiantes de introducción a la comunicación académica.,»19 7 2018. [En línea]. Available: http://dspace.casagrande. edu.ec:8080/bitstream/ucasagrande/1379/1/Tesis1575VELu.pdf. [Último acceso: 223 2021].

[11]H. Ortiz, Plataforma Para el Control del uso de Procesos de Enseñanza, Cuba: Universidad de cienfuegos, 2009.

[12]Raffino, Maria Estela, «Recursos Didácticos,» Argentina, 119 2020. [En línea]. Available: https://concepto. de/recursos-didacticos/. [Último acceso: 173 2021].

[13]Nell, David; Cortez, Liliana;, Procesos y fundamentos de la investigación científica, Machala-Ecuador: UTMACH, 2017.

[14] Rey Sanchez , Ernesto; Escalera Gamiz, Águeda, «El portafolio digital un nuevo instrumento de evaluación,» DIM, vol. 21, nº 2, p. 10, 2011.

\section{RESUMEN CURRICULAR}

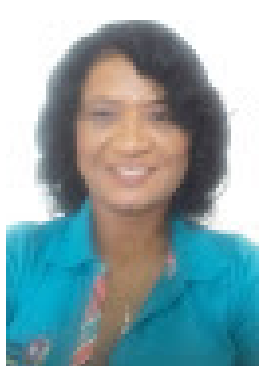

Dolores Montaño Flores, de nacionalidad ecuatoriana, ingeniera en sistemas $y$ computación y magister en tecnologías para la gestión y práctica docente, actual docente contratada en el área de TICS de la Universidad Técnica Luís Vargas Torres. 